\title{
Global statistical tests for clustered earthquake pattern discovery in Italy
}

\author{
Renata Rotondi, Elisa Varini \\ Consiglio Nazionale della Ricerche, Istituto di Matematica Applicata e Tecnologie Informatiche, \\ Milano, Italy
}

\begin{abstract}
It is a widely shared opinion that not only secondary earthquakes (aftershocks) but also main earthquakes tend to occur in time-space clusters. The importance of this assumption requires the application of statistical tools to objectively evaluate its coherence with the reality at different scales of size-space-time. Global tests allow us to select the data sets with significant space-time clustering in order to perform more in-depth analyses to detect cluster locations. According to different fixed magnitude thresholds, we perform two global statistical tests, the Knox test and the Jacquez test, based on the space-time distance between pairs of earthquakes under the null hypothesis of uniform distribution in time and space, and evaluate the significance of the possible clusters. We analyze subsets of historical Italian earthquakes drawn from the Parametric Catalog of Italian Earthquakes (CPTI04) with magnitude thresholds 4.5, 5.3 and 6.0, associated with the composite seismogenic sources of the Database of Individual Seismogenic Sources. Each subset is related to one of the eight tectonically homogeneous macroregions in which the Italian territory has been divided. Significant space-time clustering is found for all sets with a magnitude threshold of 4.5. This tendency decreases drastically or disappears when the cut off rises to 5.3, with the exception of two macroregions located in the Eastern Alps and the Calabrian Arc, respectively, where evidence of space-time interaction may refer to stress transfer among consecutive or adjacent faults. The link between clustering effect and tectonic behavior could guide the choice of different stochastic point processes to model the seismic activity.
\end{abstract}

Correspondence: Elisa Varini, CNR-IMATI, via Bassini 15, 20133 Milano, Italy. Tel. +39.02 .23699527 - Fax: +39.02 .23699538 .

E-mail: elisa@mi.imati.cnr.it

Key words: long-term earthquake clustering, global statistical tests, earthquake catalog statistics.

Acknowledgments: the authors thank the DISS Working Group, especially Roberto Basili, for the partition of the Italian territory in tectonically homogeneous macroregions, and for the association of earthquakes from the CPTI04 catalog with the composite seismogenic sources of the DISS database (rel. 3.0.2). Sincere thanks are also due to both reviewers for their helpful comments and suggestions.

Received for publication: 16 December 2011.

Revision received: 13 April 2012.

Accepted for publication: 18 April 2012.

This work is licensed under a Creative Commons Attribution NonCommercial 3.0 License (CC BY-NC 3.0).

(C) Copyright R. Rotondi and E. Varini, 2012

Licensee PAGEPress, Italy

Research in Geophysics 2012; 2:e9

doi:10.4081/rg.2012.e9

\section{Introduction}

Historical seismic catalogs from all around the world show the occurrence of couples, or also multiplets, of large earthquakes. ${ }^{1}$ Many scientists have raised the key question of whether strong events are independent or if they can be considered independent because they have negligible interactions or, very differently, if they tend to occur in space-time clusters. The latter hypothesis is now the most supported: it constitutes a challenge for the most widely used long-term time-independent forecasting models and reopens the complicated question as to the universality of the physical law for earthquake generation at different scales of magnitude, space and time.

Furthermore, this assumption has affected the preferential choice of stochastic models, such as self-exciting (epidemic) models in the studies on seismic hazard, i.e. models that imply the abrupt increase in the occurrence probability after a shock and the subsequent exponential decrease, without the desirable increase before a forthcoming event.

Lombardi and Marzocchi ${ }^{2}$ observed that, worldwide, $M_{S} 7.0+$ earthquakes tend to cluster in time and space, with features similar to smaller events. They, therefore, proposed an Epidemic-Type AftershockSequence (ETAS) model with time-dependent background rate, named NETAS, in order to describe the temporal distribution of the strong earthquakes collected in the worldwide seismic catalog. Kagan and Jackson $^{3}$ claimed that the major feature of earthquake occurrence is time-space clustering, both short- and long-term. On the basis of this conjecture they then developed a worldwide short- and long-term earthquake forecast based on smoothed maps of past seismicity and the assumption of spatial and temporal clustering. ${ }^{3,4}$ In the last report by the International Commission on Earthquake Forecasting for Civil Protection, ${ }^{5}$ the class of time-dependent models based on the long-term space-time earthquake clustering (i.e. self-exciting models) is recognized as being more promising for long-term forecasting than the family of models where, after a strong earthquake, some time should elapse to build enough stress for another rupture along the same fault. In this paper, the historical Italian seismicity is considered. No long-term forecasting model will be chosen a priori. Our aim is to check the statistical plausibility of the assumption underlying the above-mentioned models, that are considered as being the best stochastic models. Suitable statistical tests will be applied to evaluate whether damaging earthquakes that were recorded in different tectonic regions in Italy are likely to occur in space-time clusters. Many statistical tests for space-time clustering are available in the literature, especially in epidemiological applications. ${ }^{6}$ We consider some of the most widely used, namely the Knox test and the Jacquez test. Both of them are global tests, i.e., they have been designed to provide a single (global) statistic characterizing any deviation of a map pattern from the null hypothesis of space-time randomness. In the future, other types of tests could be used to detect size and location of clusters: focused and local tests. The former evaluate whether clustering occurs around particular foci, while the latter are performed to detect clustering when there is no a priori idea of where and how large the clusters may be. Once a global test is 
carried out, a deeper analysis could be performed in the following way: if the outcome of the global test reveals no significant deviation from a random space-time pattern, local tests can uncover isolated hot spots of increased activity. Otherwise, local tests can be used to distinguish between two possible situations: local statistics are quite similar throughout the region, indicating a relatively homogeneous region; or local outliers are detected and the global statistic results are mainly affected from them.

This paper only considers global tests because the aim is to test the hypothesis of random size-space-time distribution of the historical earthquakes in Italy. Later, the criteria leading to the partition of Italy into the eight seismic macroregions will be examined and the two wellknown global tests due to Knox and Jacquez will be described. ${ }^{7,8}$ Results of both tests are then reported and compared for all the considered sets of earthquakes. Finally these results will be discussed.

\section{The Italian macroregions}

Data were collected by exploiting two primary databases: the Parametric Catalog of Italian Earthquakes (CPTI04) ${ }^{9}$ and the Database of Individual Seismogenic Sources (DISS) (version 3.0.2). ${ }^{10}$

CPTI04 stores all earthquakes that have occurred in Italy or in neighboring countries and felt in Italy with significant intensity. It exploits all sources of historical data available in previous historical catalogs and published scientific works. CPTI04 includes the historical records up to the year 2002 and provides a homogeneous determination of the moment magnitude $M_{w}$ and of surface-wave magnitude $M_{S}$. The catalog is declustered by removing events that occurred within 90 days and 30 $\mathrm{km}$ of a large event that has been identified as the main shock in a seismic sequence.

Database DISS is a large repository of geological, tectonic and activefault data for Italy and the surrounding areas, compiled from first-hand experience of its authors and from a large number of references. DISS stores two main categories of fully-parameterized crustal fault sources: Individual Seismogenic Sources (ISS) and Composite Seismogenic Sources (CSS), both considered to be capable of releasing earthquakes of $M_{w} 5.5$ or larger. In most cases, the ISS represent the preferred source solutions of well known large earthquakes in the past that ideally ruptured the fault from end to end (i.e. a fault segment). A CSS is essentially an inferred active structure based on regional surface and subsurface geological data that are exploited in order to identify and map complete fault systems. The DISS version 3.0.2 includes 81 seismogenic sources, most of which are located in Italy (Figure 1).

Furthermore, Basili (R Basili, personal communication, 2008) subdivided the Italian territory into eight large zones (Figure 1). These were named macroregions (MR) since they are larger than the usual size of zones in zonation models used for standard seismic hazard assessment in Italy. To draw the macroregions, zones from the seismic zonation ZS911 are aggregated on the basis of their common tectonic characteristics and their boundaries are refined in order to make them include seismogenic sources that belong to the same tectonic domain.

Since the seismogenic sources do not have their seismic history explicitly stored in the database DISS, we asked the authors of the DISS to associate the historical earthquakes of the CPTI04 catalog with their most likely seismogenic source. In order to do this, they first identified the earthquakes that are associated with an ISS on the basis of geological/geophysical studies and associated them with the CSS containing it. The remaining earthquakes were then manually associated with their parent CSS by considering their distance. It is important to remember that historical offshore events are inevitably located near the coast and can be mistaken for real coastal events.

Through this assignment procedure, eight data sets were obtained in which each earthquake is characterized by its time of occurrence, magnitude, epicentral latitude, epicentral longitude (derived from CPTI04) and macroregion of membership (including the CSS it is associated with).

As missing data could heighten the clustering tendency in the recent seismicity, at first we consider only events that took place after 1600 ; the influence of the incompleteness of the catalog in the present context will then be examined in more detail.

Even though data are derived from a declustered catalog, some pairs of strong events that are close in both space and/or time are observed. The aim was to test statistically whether this earthquake coupling is due to chance against the hypothesis that a macroregion is prone to release pairs (or also multiplets) of strong events. Furthermore, in order to check if the space-time interaction between events is maintained at different magnitude levels, data of each macroregion are analyzed by choosing three magnitude thresholds: $4.5,5.3$ and 6.0 , when data are sufficient. Table 1 reports the number of data in the considered sets in comparison with all data available in the catalog.

\section{Global statistical tests for space-time clustering}

Let $n$ be the number of events recorded in a region, $\tau_{1}, \tau_{2}, \ldots, \tau_{n}$, their times of occurrence and $\left(x_{1} y_{1}\right), \ldots,\left(x_{n} y_{n}\right)$, the spatial coordinates of their epicenters. The spatial distance between two events $i$ and $j$ is denoted by the Euclidean distance $d_{i j}^{S}=\sqrt{\left(x_{i}-x_{j}\right)^{2}+\left(y_{i}-y_{j}\right)^{2}}$ and

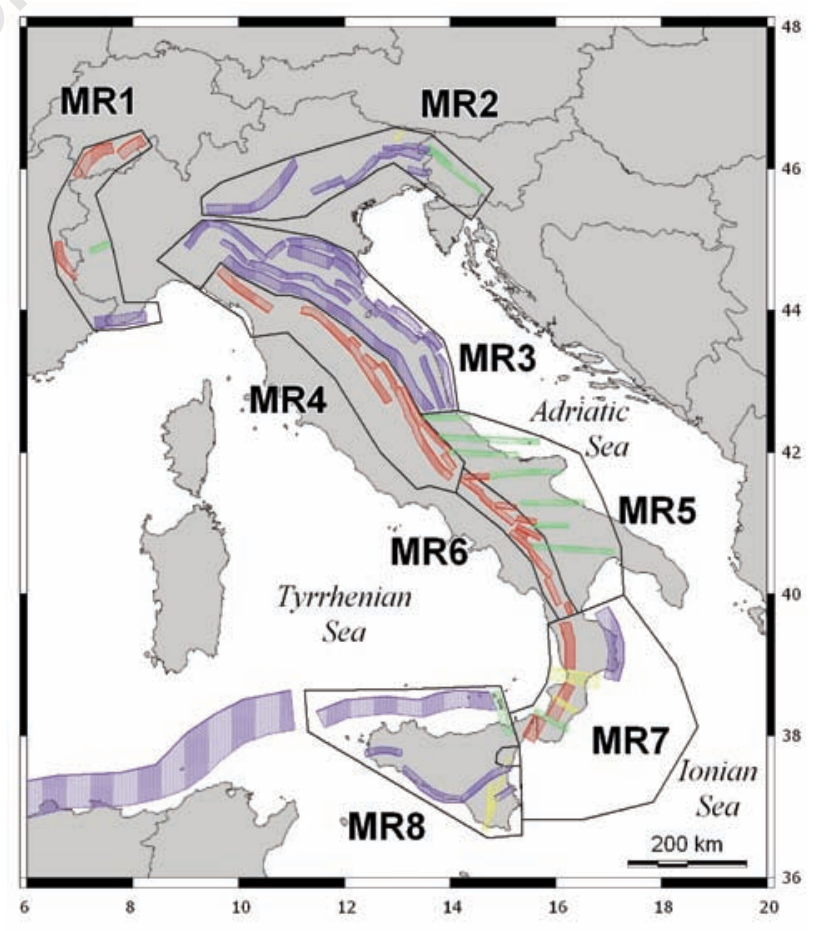

Figure 1. Map of the Composite Seismogenic Sources from the Database of Individual Seismogenic Sources, version 3.0.2 (DISS Working Group, 2007), classified according to faulting mechanism. Colored area: vertical projection of fault to ground surface, where colors identify normal (red), reverse (blue), rightlateral strike-slip (green) or left-lateral strike-slip (yellow) fault mechanisms. Black polygons are the eight macroregions under consideration. 
their time distance by the Manhattan distance ${d^{T}}^{T}=\left|\tau_{\mathrm{i}}-\tau_{\mathrm{j}}\right|$, for all $i, j$ $\in\{1,2, \ldots, n\}$.

The Euclidean distance does not take into account the curvature of the Earth because of the relatively small size of the macroregions, provided that the origin of the reference Cartesian coordinate systems is chosen near the macroregions. The null hypothesis $\mathbf{H}_{0}$ of both the Knox and the Jacquez test is:

$\mathbf{H}_{0}$ : the temporal distances between pairs of observations are independent of the spatial distances,

against the alternative hypothesis $\mathbf{H}_{1}$ : there is space-time interaction between events (i.e. $\mathbf{H}_{1}$ : not $\mathbf{H}_{0}$ ).

Both tests aim to count the observed number $\mathbf{T}$ of pairs of events close in both space and time; if this number is significantly big, there is evidence of space-time clustering. Count T represents the test statistic, defined as follows:

$$
\mathrm{T}=\frac{1}{2} \sum_{i=1}^{n} \sum_{j=1}^{n} a_{i j}^{S} a_{i j}^{T}
$$

where $a^{S}{ }_{i j}$ and $a^{T}{ }_{i j}$ are the measurements of closeness in space and in time, respectively, for all pairs $i, j$ of events. Later on, $t$ will denote the realization of $\mathrm{T}$.

In the Knox test, two events are defined to be close in space (equation 2) if they occur within a pre-specified critical space limit $\delta_{S}$; similarly, they are close in time (equation 3 ) if they occur within a pre-specified critical time limit $\delta_{T}$.

$$
\begin{aligned}
a_{i j}^{S} & = \begin{cases}1 & \text { if } i \neq j \text { and } d_{i j}^{S}<\delta_{S} \\
0 & \text { otherwise }\end{cases} \\
a_{i j}^{T} & = \begin{cases}1 & \text { if } i \neq j \text { and } d_{i j}^{T}<\delta_{T} \\
0 & \text { otherwise }\end{cases}
\end{aligned}
$$

Under the null hypothesis of no space-time interaction, the distribution of $\mathrm{T}$ has the following mean and variance: ${ }^{12}$

$$
\begin{aligned}
E\left(\mathrm{~T} \mid \boldsymbol{H}_{0}\right) & =\frac{N_{1 S} N_{1 T}}{N}, \\
V\left(\mathrm{~T} \mid \boldsymbol{H}_{0}\right) & =\frac{N_{1 S} N_{1 T}}{N}+\frac{4 N_{2 S} N_{2 T}}{n(n-1)(n-2)}-\left(\frac{N_{1 S} N_{1 T}}{N}\right)^{2}+ \\
& +\frac{4\left[N_{1 S}\left(N_{1 S}-1\right)-N_{2 S}\right]\left[N_{1 T}\left(N_{1 T}-1\right)-N_{2 T}\right]}{n(n-1)(n-2)(n-3)}
\end{aligned}
$$

where $N=\frac{n(n-1)}{2}$ is the total number of pairs of events, $N_{1 S}=\frac{1}{2} \sum_{i=1}^{n} \sum_{j=1}^{n} a_{i j}^{s}$

the number of pairs close in space, $N_{1 T}=\frac{1}{2} \sum_{i=1}^{n} \sum_{j=1}^{n} a_{i j}^{T}$ the number of pairs

close in time, $N_{2 s}=\frac{1}{2} \sum_{i=1}^{n} \sum_{j=1}^{n} \sum_{k=1}^{n} a_{i j}^{s} a_{i k}^{s}$ the number of distinct pairs of $j$ and $k$

events both close in space to another event and $N_{2 T}=\frac{1}{2} \sum_{t=1}^{n} \sum_{j=1}^{n} \sum_{k=1}^{n} a_{i j}^{T} a_{i k}^{T}$

similarly for time. The mean $E\left(\mathrm{~T} \mid \mathrm{H}_{0}\right)$ represents the expected number of pairs close in both space and time by assuming a random pattern.

The distribution of $\mathbf{T}$ under the null hypothesis (or null distribution of $\mathbf{T}$ ) would be necessary to obtain an exact evaluation of the P-value
Table 1. Number $\mathbf{n}$ of earthquakes of moment magnitude not smaller than 4.5, 5.3 and 6.0, associated with each macroregion, reported in the entire Parametric Catalog of Italian Earthquakes (CPTI04) catalog (all) and that occurred after $1600(\mathrm{~T} \geq 1600)$.

\begin{tabular}{lcccccc} 
& \multicolumn{2}{c}{$M_{w} \geq 4.5$} & \multicolumn{2}{c}{$M_{w} \geq 5.3$} & \multicolumn{2}{c}{$M_{w} \geq 6.0$} \\
& all & $T \geq 1600$ & all & $T \geq 1600$ & all & $T \geq 1600$ \\
MR1 & 119 & 108 & 23 & 21 & 2 & 2 \\
MR2 & 249 & 215 & 33 & 26 & 8 & 4 \\
\hline MR3 & 316 & 257 & 70 & 50 & 2 & 2 \\
MR4 & 440 & 383 & 73 & 45 & 19 & 9 \\
\hline MR5 & 96 & 89 & 23 & 17 & 9 & 6 \\
MR6 & 102 & 90 & 31 & 20 & 17 & 9 \\
\hline MR7 & 205 & 190 & 50 & 43 & 19 & 14 \\
MR8 & 149 & 137 & 30 & 26 & 7 & 4 \\
\hline Total & 1676 & 1469 & 333 & 248 & 83 & 50 \\
\hline
\end{tabular}

MR, macroregions.

(or observed significant level) for the statistical test: when the P-value is significantly small $(<0.05)$, the null hypothesis is rejected in favor of the alternative one of space-time clustering.

For the moment, the distribution of $\mathbf{T}$ is unknown. In 1964, Knox ${ }^{13}$ conjectured that $\mathrm{T}$ is approximately Poisson distributed. Then in 1966 Barton and David ${ }^{12}$ showed that in many cases this is true, for example, when $N_{1 S}$ and $N_{1 T}$ are small compared to $N$ so that the variance of $\mathrm{T}$ is approximately equal to its expected value. Given this, the P-value is obtained by one of the following:

(i) when $N$ is large and $N_{1 S}, N_{1 T}$ are so small with respect to $N$ (about $<5 \% N$ ), i.e. $E\left(\mathrm{~T} \mid \mathrm{H}_{0}\right)$ is roughly equal to $V\left(\mathrm{~T} \mid \mathrm{H}_{0}\right)$ by equation (4) and its value is less than 20 , then the test statistic $\mathbf{T}$ under $\mathbf{H}_{0}$ is assumed to have Poisson distribution with parameter $E\left(\mathrm{~T} \mid \mathbf{H}_{0}\right)$. Consequently, given the observed value $\mathrm{T}=t$, the $\mathrm{P}$-value is estimated by the so-called mid-P-value:?

$$
p-\text { value }=1-\sum_{k=0}^{t} \frac{E\left(\mathcal{T} \mid \mathbf{H}_{0}\right)^{k}}{k !} e^{-E\left(T \mid \mathbf{H}_{0}\right)}+\frac{1}{2} \frac{E\left(\mathcal{T} \mid \mathbf{H}_{0}\right)^{t}}{t !} e^{-E\left(T \mathbf{H}_{0}\right)} .
$$

(ii) when $N$ is large and $E\left(\mathrm{~T} \mid \mathbf{H}_{0}\right)$ is larger than 20 , then $\mathbf{T}$ under $\mathbf{H}_{0}$ is assumed to be Normal distributed with mean $E\left(\mathrm{~T} \mid \mathrm{H}_{0}\right)$ and variance $V\left(\mathrm{~T} \mid \mathbf{H}_{0}\right)$ and the $\mathrm{P}$-value is given by:

$p-$ value $\approx 1-\phi\left(\frac{t-E\left(\mathcal{T} \mid \mathbf{H}_{0}\right)}{\sqrt{V\left(\mathcal{T} \mid \mathbf{H}_{0}\right)}}\right)$,

where $\Phi(\cdot)$ is the standard Normal distribution function. The rationale lies in the asymptotic Normal approximation to Poisson distribution.

(iii) otherwise, Monte Carlo hypothesis testing can be used: a number $R$ of simulated data sets is obtained by randomly permuting the observed times $\tau_{1}, \tau_{2}, \ldots, \tau_{n}$ among the fixed observed spatial locations $\left(x_{1}, y_{1}\right), \ldots,\left(x_{n}, y_{n}\right)$ (or vice versa). For each of the $R$ simulated data sets, equation (1) provides the value $t_{v}$ of the statistics under the null hypothesis. Finally, the simulated P-value (denoted by M P-value) is obtained by determining the proportion of simulated $t_{v}$ 's that exceeds the observed value $t$ of T:

$$
\text { M p-value }=\frac{1+\sum_{\gamma=1}^{R} I\left(t_{v} \geq t\right)}{R+1},
$$


where $I(\cdot)$ is the indicator function. A number $R=1000$ is hereinafter chosen. Although this Monte Carlo method is theoretically applicable to any case, it may have a significant cost in terms of computational time for a large value of $N$. Therefore, we have limited its use where $N$ is small enough so that the simulation time does not exceed $10 \mathrm{~min}$.

The Jacquez test is based on the same equation (1), counting the number of observed pairs that are close in both time and space; but, differently from the Knox test, the measure of closeness is defined by the $k$-nearest neighbors:

$$
\begin{aligned}
a_{i j}^{s} & = \begin{cases}1 & \text { if } i \text { and } j \text { are the } k \text {-nearest neighbours in space } \\
0 & \text { otherwise }\end{cases} \\
a_{i j}^{T} & = \begin{cases}1 & \text { if } i \text { and } j \text { are the } k \text {-nearest neighbours in time } \\
0 & \text { otherwise }\end{cases}
\end{aligned}
$$

where $k$ is a pre-specified integer number. The P-value of the Jacquez test is simulated by the Monte Carlo hypothesis testing because the null distribution of the test statistic $\mathbf{T}$ is unknown; nevertheless, the

Table 2. Summary of the observed spatial and temporal distances for all the considered data sets: minimum distance (min), first quartile (Q1), median distance (Q2), third quartile (Q3), maximum distance (max) are reported besides the total number $\mathrm{N}$ of pairs of earth-

\begin{tabular}{|c|c|c|c|c|c|c|c|c|}
\hline & $M_{w}$ cut off & N. pairs & Distance & Min & Q1 & Q2 & Q3 & Max \\
\hline MR1 & 5.3 & $\begin{array}{c}210 \\
1\end{array}$ & $\begin{array}{l}\text { Km } \\
\text { Years } \\
\text { Km } \\
\text { Years } \\
\text { Km } \\
\text { Years }\end{array}$ & $\begin{array}{c}0 \\
0.0214 \\
1.89 \\
0.341 \\
268.85 \\
58.92\end{array}$ & $\begin{array}{c}57.68 \\
26.09 \\
70.30 \\
35.97 \\
268.85 \\
58.92\end{array}$ & $\begin{array}{c}121.39 \\
55.62 \\
141.83 \\
74.14 \\
268.85 \\
58.92\end{array}$ & $\begin{array}{c}198.32 \\
102.16 \\
234.41 \\
131.21 \\
268.85 \\
58.92\end{array}$ & $\begin{array}{c}294.84 \\
379.08 \\
283.72 \\
316.10 \\
268.85 \\
58.92\end{array}$ \\
\hline MR2 & 5.3 & $\begin{array}{c}23005 \\
325 \\
6\end{array}$ & $\begin{array}{l}\text { Km } \\
\text { Years } \\
\text { Km } \\
\text { Years } \\
\text { Km } \\
\text { Years }\end{array}$ & $\begin{array}{c}0 \\
0.00274 \\
1.005 \\
0.0354 \\
51.536 \\
25.879 \\
\end{array}$ & $\begin{array}{c}53.99 \\
29.29 \\
36.44 \\
45.37 \\
58.85 \\
102.86 \\
\end{array}$ & $\begin{array}{c}115.95 \\
67.93 \\
110.10 \\
93.65 \\
124.59 \\
165.40\end{array}$ & $\begin{array}{l}189.68 \\
125.37 \\
163.70 \\
152.46 \\
184.56 \\
255.32 \\
\end{array}$ & $\begin{array}{l}395.79 \\
395.48 \\
357.80 \\
303.13 \\
200.09 \\
281.20\end{array}$ \\
\hline MR3 & $\begin{array}{l}4.5 \\
5.3 \\
6.0\end{array}$ & $\begin{array}{c}32896 \\
1225 \\
1\end{array}$ & $\begin{array}{l}\text { Km } \\
\text { Years } \\
\text { Km } \\
\text { Years } \\
\text { Km } \\
\text { Years }\end{array}$ & $\begin{array}{c}0 \\
0.000436 \\
2.462 \\
0.122 \\
44.983 \\
40.109\end{array}$ & $\begin{array}{c}68.09 \\
29.94 \\
64.15 \\
45.21 \\
44.983 \\
40.109\end{array}$ & $\begin{array}{c}130.75 \\
68.27 \\
124.10 \\
100.51 \\
44.983 \\
40.109\end{array}$ & $\begin{array}{l}211.93 \\
143.22 \\
189.93 \\
179.10 \\
44.983 \\
40.109\end{array}$ & $\begin{array}{l}610.23 \\
400.36 \\
529.67 \\
398.23 \\
44.983 \\
40.109\end{array}$ \\
\hline MR4 & $\begin{array}{l}4.5 \\
5.3\end{array}$ & $\begin{array}{c}73153 \\
990 \\
36\end{array}$ & $\begin{array}{c}\mathrm{Km} \\
\text { Years } \\
\mathrm{Km} \\
\text { Years } \\
\mathrm{Km} \\
\text { Years }\end{array}$ & $\begin{array}{c}0 \\
0.00166 \\
4.223 \\
0.0233 \\
12.057 \\
0.0513\end{array}$ & $\begin{array}{l}65.76 \\
24.98 \\
43.87 \\
48.37 \\
68.08 \\
59.59\end{array}$ & $\begin{array}{c}139.89 \\
60.85 \\
99.22 \\
101.11 \\
125.14 \\
168.52\end{array}$ & $\begin{array}{l}241.13 \\
132.96 \\
179.50 \\
169.66 \\
221.52 \\
261.59\end{array}$ & $\begin{array}{l}507.96 \\
401.39 \\
471.49 \\
374.41 \\
401.32 \\
357.97\end{array}$ \\
\hline MR5 & $\begin{array}{l}4.5 \\
5.3 \\
6.0\end{array}$ & $\begin{array}{l}3916 \\
136 \\
15\end{array}$ & $\begin{array}{c}\text { Km } \\
\text { Years } \\
\text { Km } \\
\text { Years } \\
\text { Km } \\
\text { Years }\end{array}$ & $\begin{array}{c}0 \\
0.0617 \\
5.427 \\
2.046 \\
28.360 \\
18.835\end{array}$ & $\begin{array}{l}48.12 \\
29.45 \\
51.31 \\
49.03 \\
48.28 \\
65.14\end{array}$ & $\begin{array}{c}89.90 \\
64.04 \\
109.31 \\
101.26 \\
82.22 \\
120.40\end{array}$ & $\begin{array}{l}157.70 \\
142.85 \\
167.29 \\
195.86 \\
136.46 \\
196.18\end{array}$ & $\begin{array}{l}463.44 \\
375.26 \\
413.82 \\
375.26 \\
185.28 \\
248.35\end{array}$ \\
\hline MR6 & $\begin{array}{l}4.5 \\
5.3 \\
6.0\end{array}$ & $\begin{array}{l}4005 \\
190 \\
36\end{array}$ & $\begin{array}{l}\text { Km } \\
\text { Years } \\
\text { Km } \\
\text { Years } \\
\text { Km } \\
\text { Years }\end{array}$ & $\begin{array}{c}0 \\
0.0274 \\
1.405 \\
3.328 \\
1.405 \\
6.258\end{array}$ & $\begin{array}{l}38.63 \\
28.19 \\
39.03 \\
52.21 \\
33.58 \\
51.88\end{array}$ & $\begin{array}{c}72.85 \\
66.49 \\
75.41 \\
106.53 \\
44.81 \\
125.02\end{array}$ & $\begin{array}{c}120.39 \\
127.25 \\
113.58 \\
172.60 \\
82.26 \\
231.26\end{array}$ & $\begin{array}{l}268.61 \\
310.65 \\
225.77 \\
310.26 \\
174.25 \\
292.47\end{array}$ \\
\hline MR7 & $\begin{array}{l}4.5 \\
5.3 \\
6.0\end{array}$ & $\begin{array}{c}17955 \\
903 \\
91\end{array}$ & $\begin{array}{l}\text { Km } \\
\text { Years } \\
\text { Km } \\
\text { Years } \\
\text { Km } \\
\text { Years }\end{array}$ & $\begin{array}{c}0 \\
0.000694 \\
4.164 \\
0.00141 \\
4.274 \\
0.00561\end{array}$ & $\begin{array}{l}50.68 \\
35.62 \\
52.61 \\
50.06 \\
42.61 \\
48.98\end{array}$ & $\begin{array}{c}83.82 \\
77.22 \\
84.07 \\
106.50 \\
61.33 \\
122.45\end{array}$ & $\begin{array}{c}125.50 \\
148.55 \\
117.74 \\
175.37 \\
94.17 \\
196.98\end{array}$ & $\begin{array}{l}293.98 \\
400.69 \\
263.84 \\
391.83 \\
183.54 \\
282.73\end{array}$ \\
\hline MR8 & 5.3 & 325 & $\begin{array}{l}\text { Km } \\
\text { Years } \\
\text { Km } \\
\text { Years } \\
\text { Km } \\
\text { Years }\end{array}$ & $\begin{array}{c}0 \\
0.00591 \\
0.724 \\
0.206 \\
6.470 \\
10.249\end{array}$ & $\begin{array}{c}68.49 \\
27.63 \\
62.12 \\
53.78 \\
107.88 \\
93.16\end{array}$ & $\begin{array}{c}109.19 \\
71.00 \\
99.62 \\
126.80 \\
148.74 \\
186.98\end{array}$ & $\begin{array}{l}157.02 \\
145.78 \\
146.03 \\
223.27 \\
186.03 \\
275.01\end{array}$ & $\begin{array}{c}346.73 \\
389.09 \\
332.352 \\
389.03 \\
195.773 \\
285.26\end{array}$ \\
\hline
\end{tabular}
quakes. 
expectation of $\mathbf{T}$ is available and is given as in equation (4).

All pairs of events that are k-nearest neighbors for a small value turn out to be $k$-nearest neighbors also for larger value of $k$. Therefore, the test statistical $\mathrm{T}$ is a cumulative measure of proximity and the number $k$ operates as a scale parameter since it determines how far we look for associations between events. When the Jacquez test for a certain $k$ value provides evidence of clustering, general information about cluster size comes from the average spatial distance $\hat{d}^{S}$ and the average temporal distance $\hat{d}^{T}{ }_{k}$ of the $k$-nearest neighbor.

This is seen as an advantage of the Jacquez test compared to the Knox test, for which the corresponding information about the cluster size is subjectively established when the user specifies the critical space and time limits. Jacquez explained this using an illustrative example: ${ }^{14}$ assuming that a geographical space is stretched equally in all directions like a rubber sheet, the geographical distance between events increases and, therefore, the number of pairs of events classified as near in space by the Knox test decreases, while the corresponding number for the Jacquez test does not change.

Therefore, the Knox and Jacquez tests are to be considered as complementary because the former is expected to perform better if the clustering process is related to the Euclidean distance while the latter should be more suitable if the clustering process is dominated by a nearest neighbors metric. In the absence of such information, they should both be applied and their results compared.

\section{Results}

The statistical tests described were applied to the seismic data sets related to the eight Italian macroregions to check whether an interaction mechanism among earthquakes would be likely to lead to events that are closer to one another in space and time than would be expected by chance.

As the critical space and time limits of the Knox test (i.e. $\delta_{S}$ and $\delta_{T}$ respectively) characterize the scale of the clustering in space and time, they should be accurately selected so as not to undermine the analysis; but we have no a priori information about them.

It seems reasonable to assume that the size of the critical limits, if any, should be compatible with the observation size. To this end, we have carried out an explorative analysis of the observed temporal $d^{T}{ }_{i j}$ and spatial $d^{S}{ }_{i j}$ distances for all data sets. Table 2 summarizes minimum, maximum, median, first and third quartiles of all the data sets. Then we repeatedly applied the Knox test for each macroregion by varying the critical parameters $\delta_{S}$ and $\delta_{T}$ in numerical sets to explore the most significant part of the empirical distribution of the corresponding distance and until the test steadily accepts the null hypothesis; this is called a multiple Knox test.

Similarly, the Jacquez test is carried out for different $k$ values so as to obtain a multiple Jacquez test: starting from small numbers, the value of $k$ is gradually increased up to satisfy the same criteria as for the Knox test.

Appendix shows the tables of the results, each concerning data from one of the eight macroregions with fixed magnitude cut off. The null hypothesis of random space-time pattern is considered rejected when the P-value is less than a significance level 0.05 (these P-values are highlighted in red in the tables of Appendix); the method i), ii) or iii) described before for assessing the P-value is also specified.

Correspondingly, Figures 2-9 display the spatio-temporal distribution of the data and provide graphical summaries of the output for the multiple tests. In these figures, the magnitude of the P-values is shown in different colors: $P$-values in shades of red are less than the significance level 0.05 denoting the rejection of $\mathrm{H}_{0}$ otherwise P-values are in shades of blue. The more intense the color is, the more the P-value differs from the chosen significance level; in this way the reader can easily deduce the test results for other typical significance levels.

A first look at the results shows an essential agreement between the Knox and Jacquez tests. All data sets with cut-off magnitude 4.5 show significant space-time clustering, but this tendency decreases drastically or disappears when the cut off rises to 5.3. This supports the conjecture that earthquake clustering is not invariant with respect to the size of the events.

Let us consider data with cut off 4.5. In the multiple Knox test for macroregions MR2, MR3, MR4 and MR7 we find space-time correlation of the critical limits $\delta_{T}$ and $\delta_{S}$ as we expected, meaning that significant clustering is found up to large $\delta_{T}$ if $\delta_{S}$ is small and vice versa. Significant Knox tests for macroregions MR1 and MR5 do not show a similar correlation. In the macroregions MR6 and MR8, significant clustering is not found for small critical limits, but only for middle values of $\delta_{T}$ and $\delta_{S}$. Care must be taken of the macroregions MR7 and MR8 because their historical offshore events could be badly located near the coast and, therefore, compromise the statistical analysis. These results are essentially confirmed by the Jacquez multiple test for all the macroregions.

Let us now consider data with cut off 5.3. No clustering effect is found in the macroregions MR4, MR5, MR6, and MR8, and only very reduced clustered activity is identified in MR1 and MR3. As far as the macroregions MR2 and MR7 are concerned, both statistical multiple tests are significant for critical values belonging to discontinuous sets. Specifically, the Knox tests on MR2 data reveal some clustering effect for small critical limits $\left(\delta_{T} \leq 2\right.$ years and $\left.\delta_{S} \leq 4 \mathrm{~km}\right)$ or for relatively large critical limits ( $5 \leq \delta_{T} \leq 70$ years and $\left.20 \leq \delta_{S} \leq 40 \mathrm{~km}\right)$. Accordingly, the average spatial distances $\hat{d}_{k}^{S}$ of the significant Jacquez tests $(k=3.6)$ are approximately $30 \mathrm{~km}$ and $60 \mathrm{~km}$. A look at the fault rupture parameters characterizing this macroregion may explain this peculiar outcome. Since MR2 is dominated by a south-verging thrust faulting mechanism, we use the regression for thrust (reverse) fault, provided by Wells and Coppersmith ${ }^{15}$ in order to estimate the downdip rupture width $W$ by the moment magnitude $M_{w}$ :

$$
\log _{10}(W)=-1.61+0.41 M_{w}
$$

When the moment magnitude $M_{w}$ is in the range from the threshold 5.3 to the maximum observed 6.6 in MR2, the average surface rupture width corresponding to an average dip of $35^{\circ}$ is expected to vary from 3 $\mathrm{km}$ to $10 \mathrm{~km}$. Given the closeness of the seismogenic sources in the eastern Alps and that their average width is approximately $10 \mathrm{~km}$, the clustering effect identified by the statistical tests may be due to the activation of adjacent seismogenic sources. In this case, local tests may help to identify possible fault interactions or to detect possible foci around which clusters typically develop after strong earthquakes.

Similar arguments can be also applied to MR1 and MR3 in order to better understand the mild evidence of space-time clustering only produced by the multiple Knox test. In particular, MR3 can be analyzed as for MR2 because of its exclusively northeast-verging thrust faulting mechanism, whereas in-depth analysis of MR1 has to be performed with caution because of the scarcity of data available for this macroregion.

For MR7, the Knox test found clustering activity at short temporal limit $\delta_{T}$ and large spatial limit $\delta_{S}$ or, specularly, at small $\delta_{S}$ and large $\delta_{T}$; the $k$ values of the significant Jacquez tests range from 6 to 13 , corresponding to an average spatial distance $\hat{d}_{k}$ of $40-60 \mathrm{~km}$ and an average temporal distance $\hat{d}^{T}{ }_{k}$ of 30-65 years related to the $k$-nearest neighbor. The large quantity of available data related to MR7 (Table 2) means the multiple Knox test can be applied also to the events with cut-off magnitude 6.0 (Appendix, Table A29 and Figure 8 bis). The multiple test sug- 

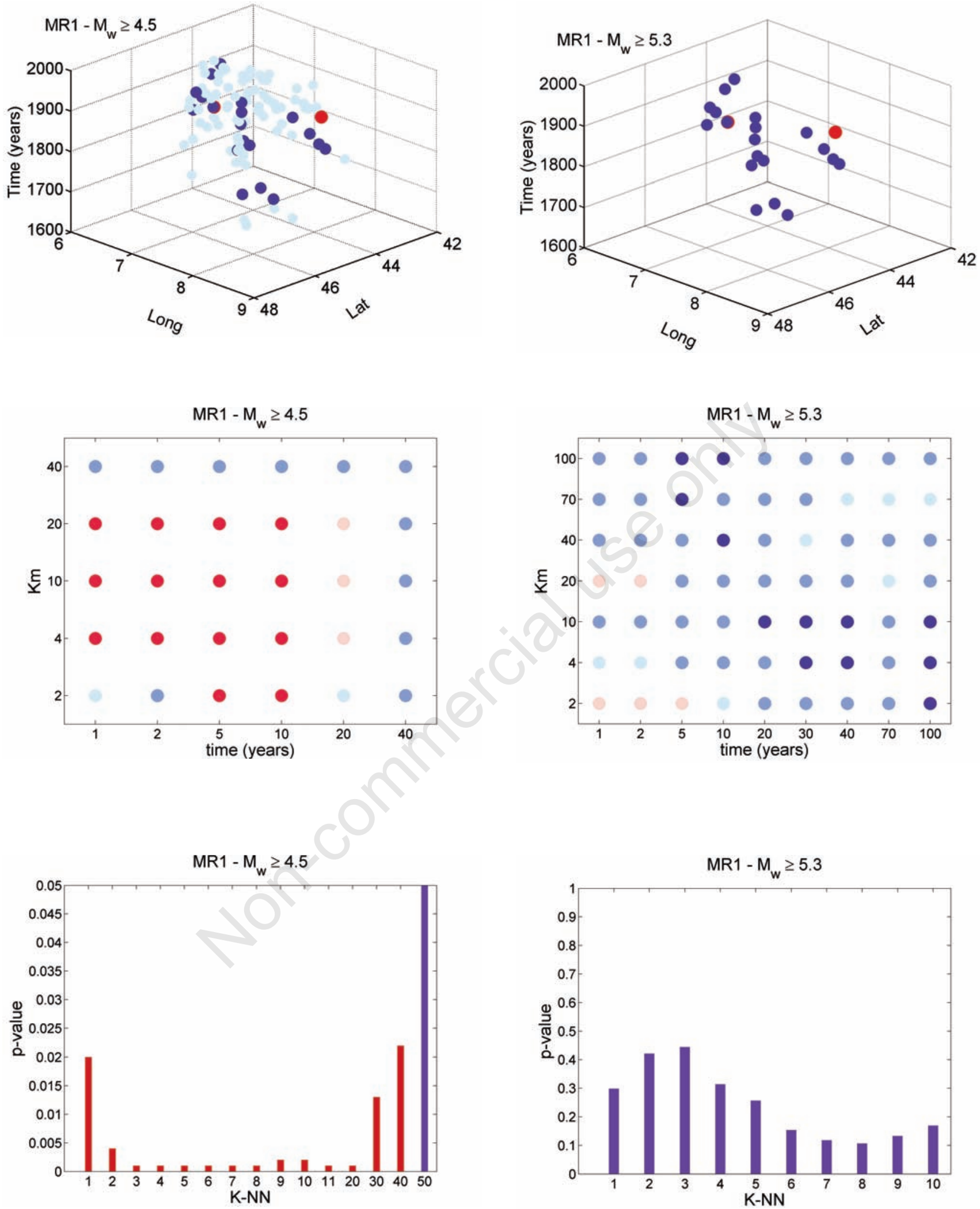

Figure 2. Results related to macroregion MR1 for data with cut-off magnitude 4.5 (left column) and 5.3 (right column), respectively. (Top) Spatio-temporal distribution of the data, where colors denote the magnitude level of the earthquakes: ${ }^{\bullet}[4.5,5.3), \bullet^{\bullet}[5.3,6.0), \bullet^{\bullet}[6.0,+\infty)$ (Middle) P-value of the Knox tests having critical limits $\delta_{T}$ in abscissa and $\delta_{S}$ in ordinate. Colors correspond to the estimated P-value: , ${ }^{\bullet}\left[0,10^{-6}\right],{ }^{\bullet}\left(10^{-6}, 0.01\right],{ }^{\circ}(0.01,0.05],{ }^{-1}(0.05,0.1],{ }^{\bullet}(0.1,0.5],{ }^{\bullet}(0.5,1]$. (bottom) Estimated P-value of the Jacquez tests versus the $k$ value: bars are blue except when $\mathrm{P} \leq 0.05$ (red). MR, macroregions. 

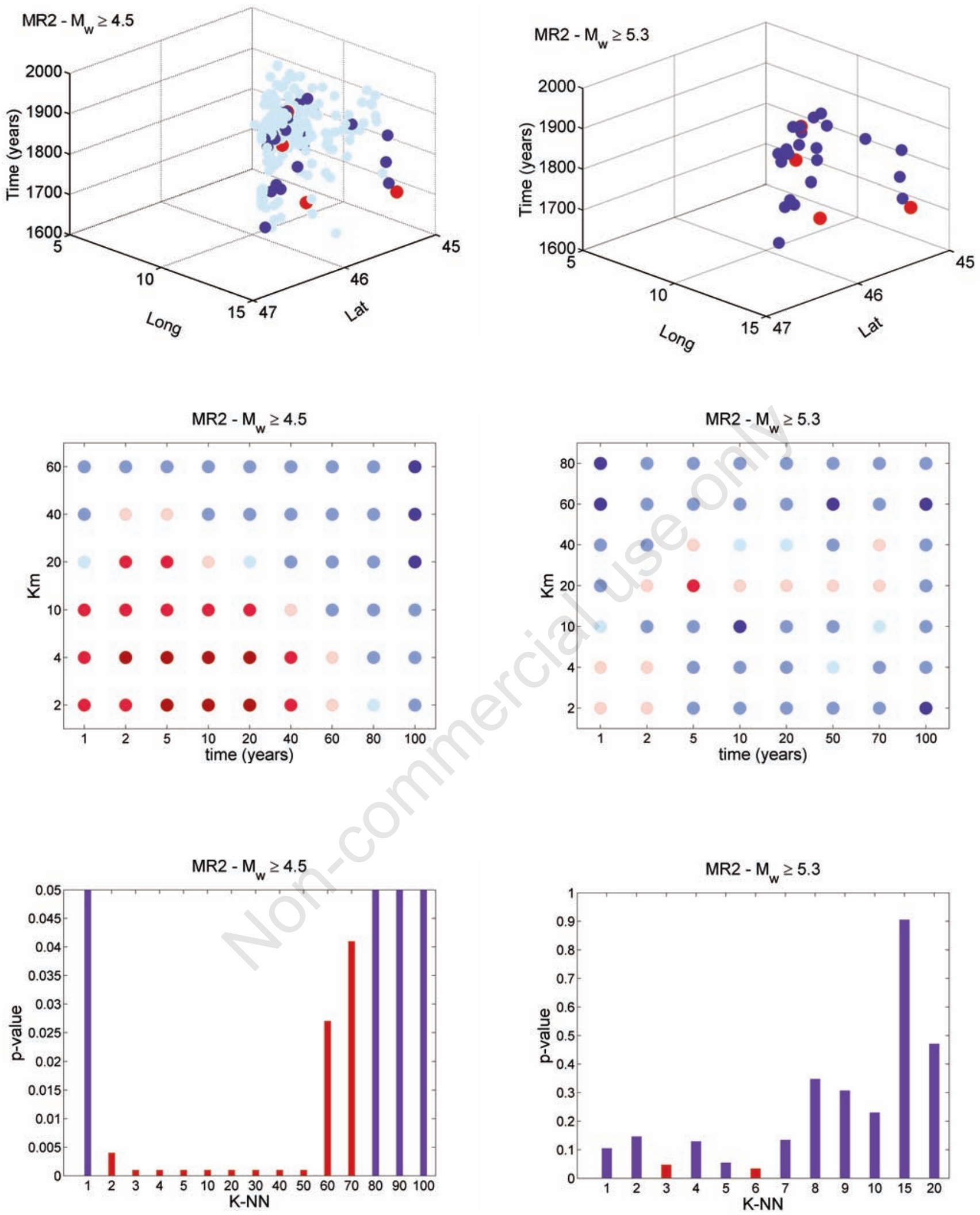

Figure 3. Results related to MR2 data with cut-off magnitude 4.5 (left) and 5.3 (right), respectively. See caption of Figure 2. MR, macroregions. 

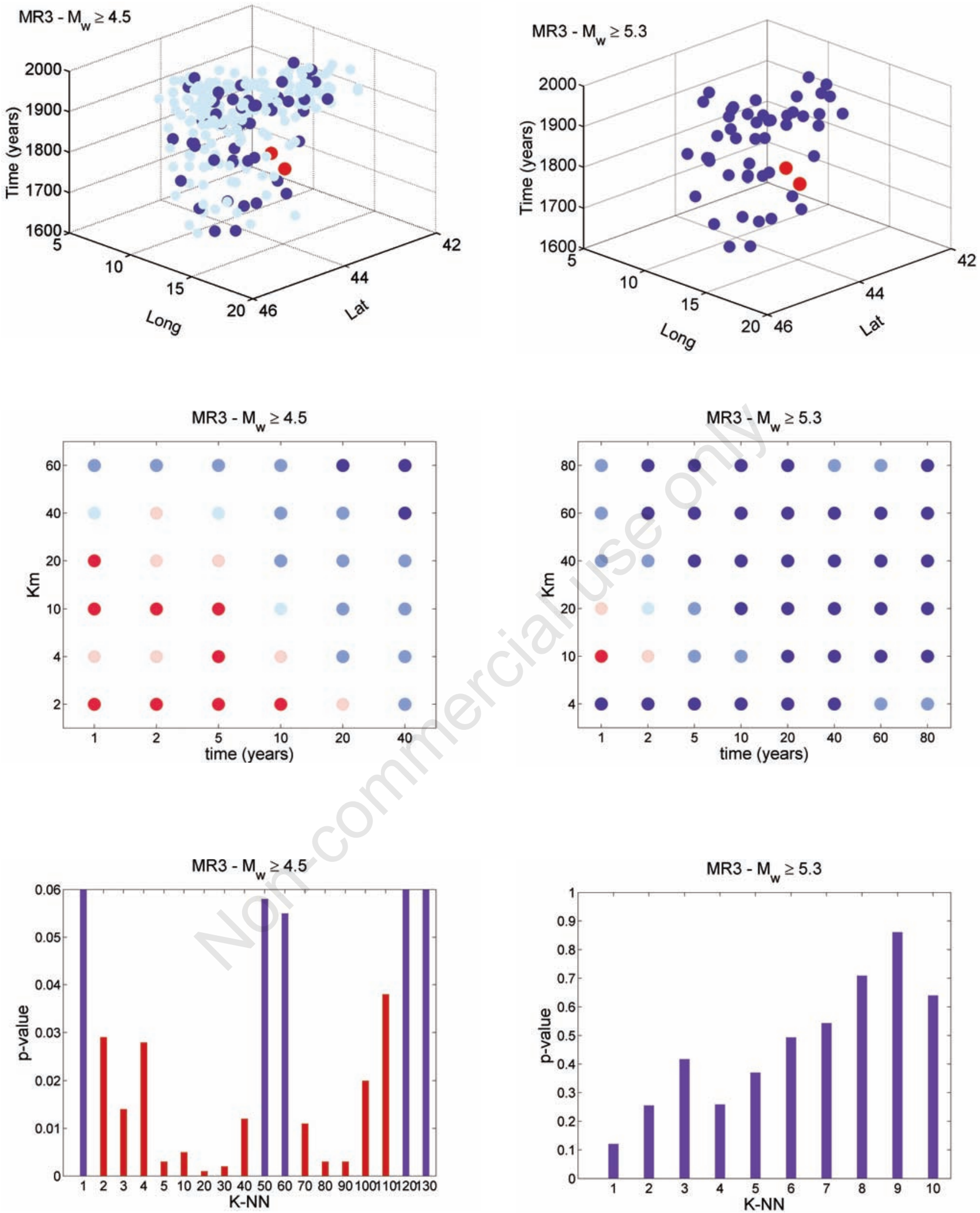

Figure 4. Results related to MR3 data with cut-off magnitude 4.5 (left) and 5.3 (right), respectively. See caption of Figure 2. MR, macroregions. 

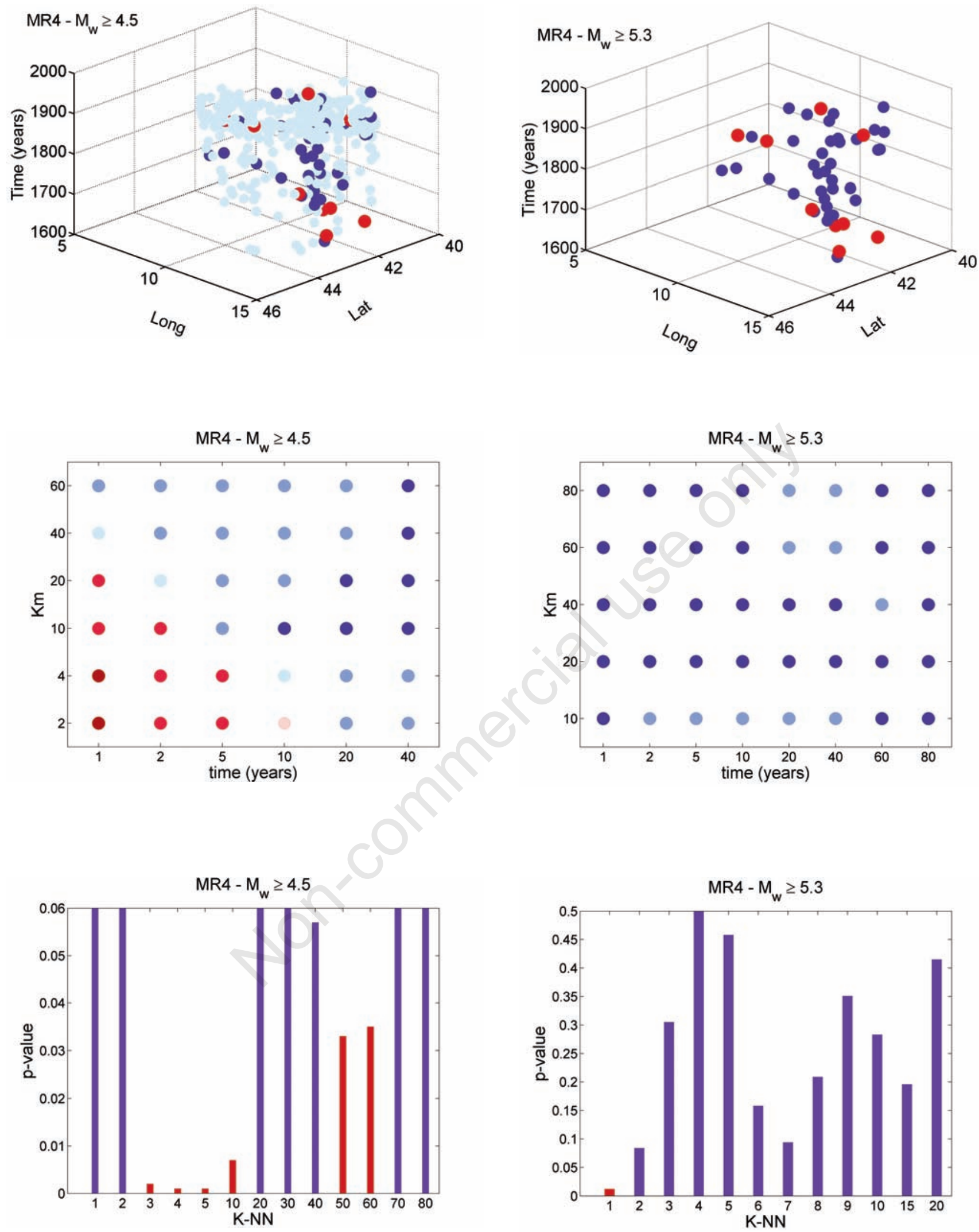

Figure 5. Results related to MR4 data with cut-off magnitude 4.5 (left) and 5.3 (right), respectively. See caption of Figure 2. MR, macroregions. 

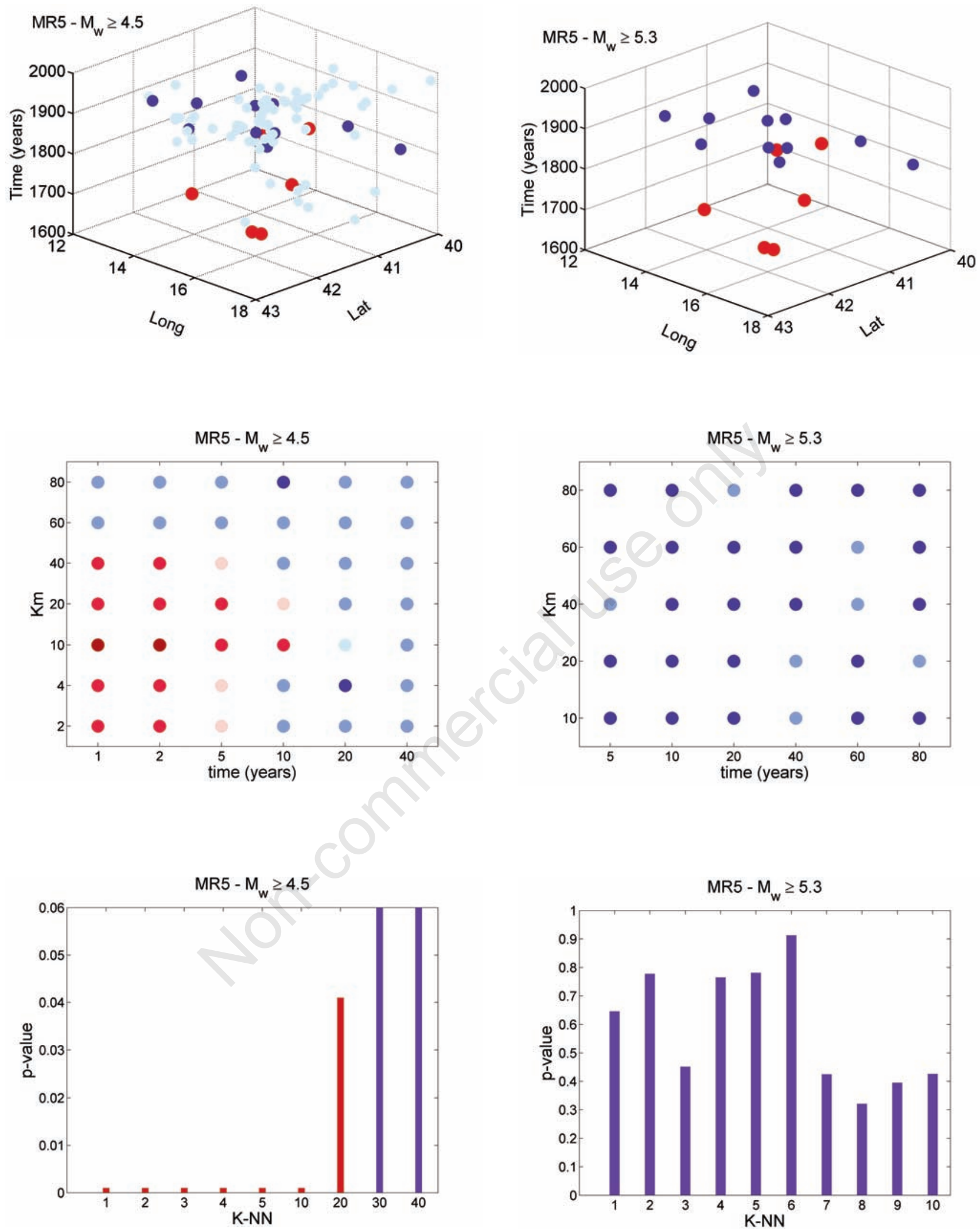

Figure 6. Results related to MR5 data with cut-off magnitude 4.5 (left) and 5.3 (right), respectively. See caption of Figure 2. MR, macroregions. 

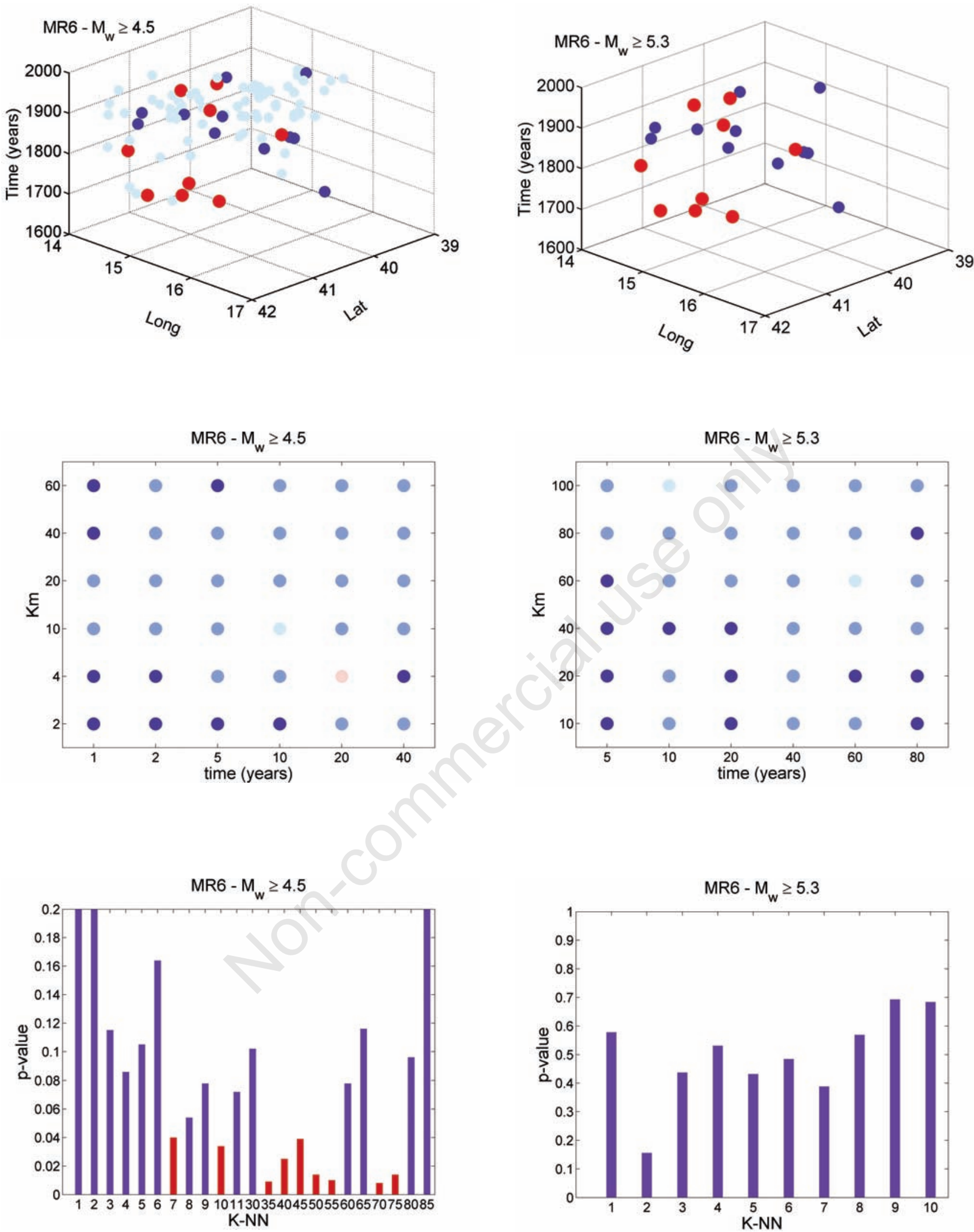

Figure 7. Results related to MR6 data with cut-off magnitude 4.5 (left) and 5.3 (right), respectively. See caption of Figure 2. MR, macroregions. 

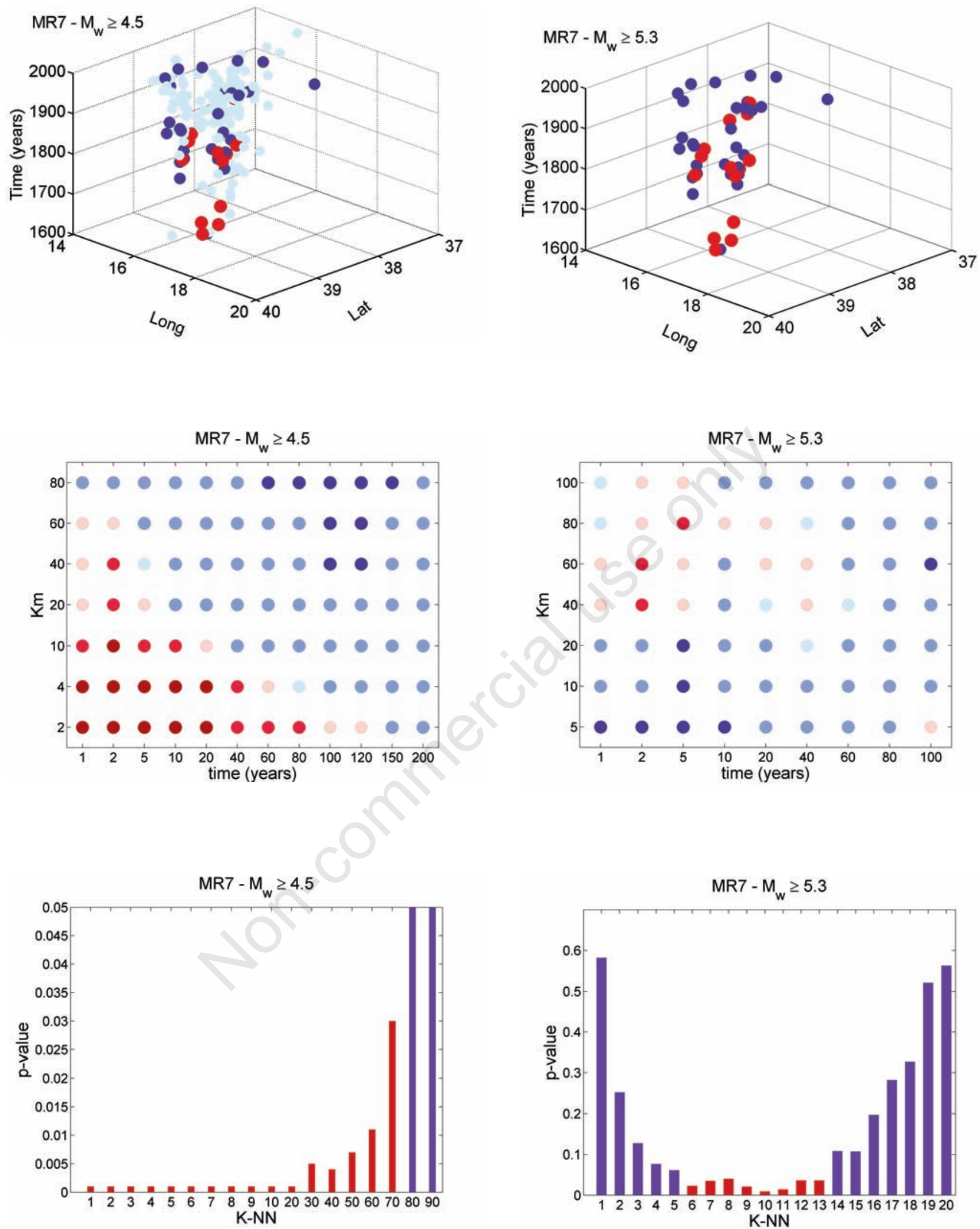

Figure 8. Results related to MR7 data with cut-off magnitude 4.5 (left), 5.3 (right) respectively. See caption of Figure 2. MR, macroregions. 
gests again that space-time clustering is observable within the same distance of $60-80 \mathrm{~km}$ and in the time interval of $10-40$ years; this additional result corroborates the alternative hypothesis for the macroregion MR7. This is particularly interesting because MR7 was hit by some of the most powerful Italian earthquakes (the second strongest earthquake with moment magnitude 7.24 occurred here on 28 December 1908) and estimates from the historical catalog give it a high level of seismicity. Furthermore, since the macroregion MR7 has a mixed fault mechanism, mainly characterized by normal faults and to a lesser extent by oblique-slip faults and thrust faults, we have chosen the regression related to all-type rupture faults: ${ }^{15}$

$$
\log _{10}(L)=-3.22+0.69 M_{w}
$$

to estimate the surface rupture length. For the moment, magnitude $M_{w}$ ranging from the threshold 5.3 and the maximum 7.24 observed in the macroregion, an expected surface rupture length from $3 \mathrm{~km}$ to $60 \mathrm{~km}$ is estimated. Comparing these values with the average size of the Calabrian seismogenic sources (approximately $30 \mathrm{~km}$ ), activation of consecutive faults is possible and may explain the outcome of the multiple tests.

Finally, to explore the influence of the possible incompleteness of the catalog on the clustering properties, we consider the time-interval completeness estimated on the historical basis for each zone of the zonation model ZS9 ${ }^{11}$ and adopted as input element of the most recent official MPS04 seismic hazard map of Italy. ${ }^{16}$

In the MPS04 elaboration, the magnitude is binned into 12 classes, from 4.76 to 7.41 , with increment equal to 0.23 . We, therefore, associate the time-interval completeness of each zone of ZS9 with the composite seismogenic sources included in it, wholly or for the most part, by adopting roughly the starting dates of the first class $M_{w} \geq 4.76$ for our data sets with magnitude threshold 4.5 and those of the third class $M_{w} \geq 5.22$ for the sets of magnitude threshold 5.3.

Considering the substantial agreement among the results provided by the two tests on the data after 1600 , only the Knox test was repeated on the new data sets. Remarkable differences were observed only for the data sets of $M_{w} \geq 4.5$ in MR2 and MR7. In particular, in MR2 the clustering effect comes down to the intervals covering 2-5 years and less than $20 \mathrm{~km}$, and in MR7 to the time intervals shorter than 20 years, with less extreme P-values in both cases.

Furthermore, again for $M_{w} \geq 4.5$, in MR1 the test rejects the randomness hypothesis only for events occurring by $10 \mathrm{~km}$ and 10 years and at about $2 \mathrm{~km}$ within time intervals of 5-10 years, in MR6 the hypothesis is no longer rejected, and in MR4 the clustering trend reaches out to 40 years at $2 \mathrm{~km}$ and 10 years at $4 \mathrm{~km}$.

On the other hand, for the threshold $M_{w} \geq 5.3$, the results remain essentially stable with only light variations in two macro-regions: in MR6 P-values corresponding to the critical limits $\delta_{S}=60 \mathrm{~km}-\delta_{T}=60$ years and $\delta_{S}=100 \mathrm{~km}-\delta_{T}=10$-20 years, already small, become significant, whereas in MR7 the null hypothesis is rejected just when the critical limits take these values: $\delta_{T}=2,40,80-100$ for $\delta_{S}=40$, and $\delta_{T}=2,5$, 20 for $\delta_{S}=60,80$. We can, therefore, conclude that these changes, although indicative of the influence of the catalog completeness on any analysis of seismicity, do not substantially modify the conclusions in the present context.

\section{Conclusions}

Global tests of the historical earthquakes that occurred in the eight tectonically homogeneous Italian macroregions lead us to the preliminary conclusion that there is clear statistical evidence of space-time clustering among events of middle magnitude. But this effect is weakened or even disappears among events of large magnitude for all the macroregions with the exception of MR2 and MR7. Some considerations about the faulting mechanisms of MR2 and MR7 suggest that statistical evidence may refer to stress transfer among consecutive or adjacent faults, i.e. the significant space-time clustering may be the result of some fault interactions within the macroregion. It should be emphasized that conclusions should be drawn with caution concerning macroregions MR7 because its historical offshore earthquakes may have been incorrectly placed near the coast and then have produced apparent significant clustering.

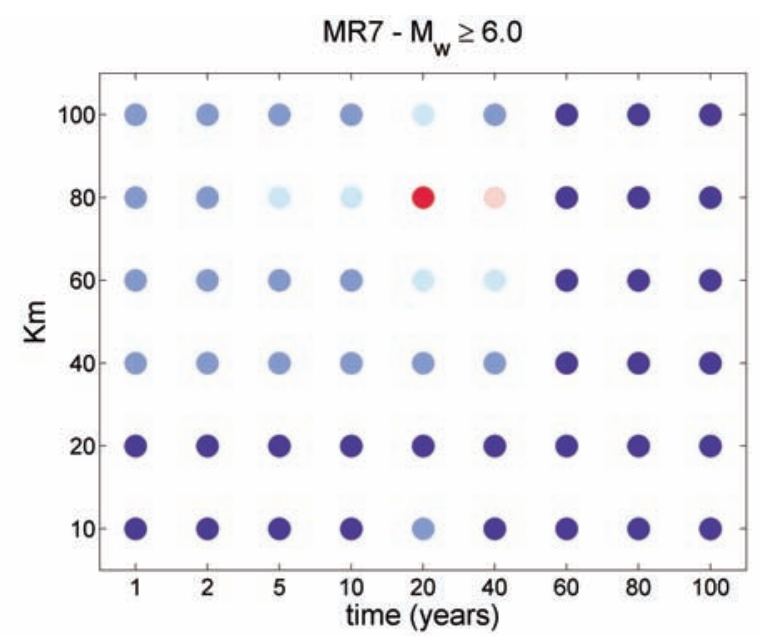

Figure 8 bis. Results related to MR7 data with cut-off magnitude 6.0. See caption of Figure 2. MR, macroregions. 

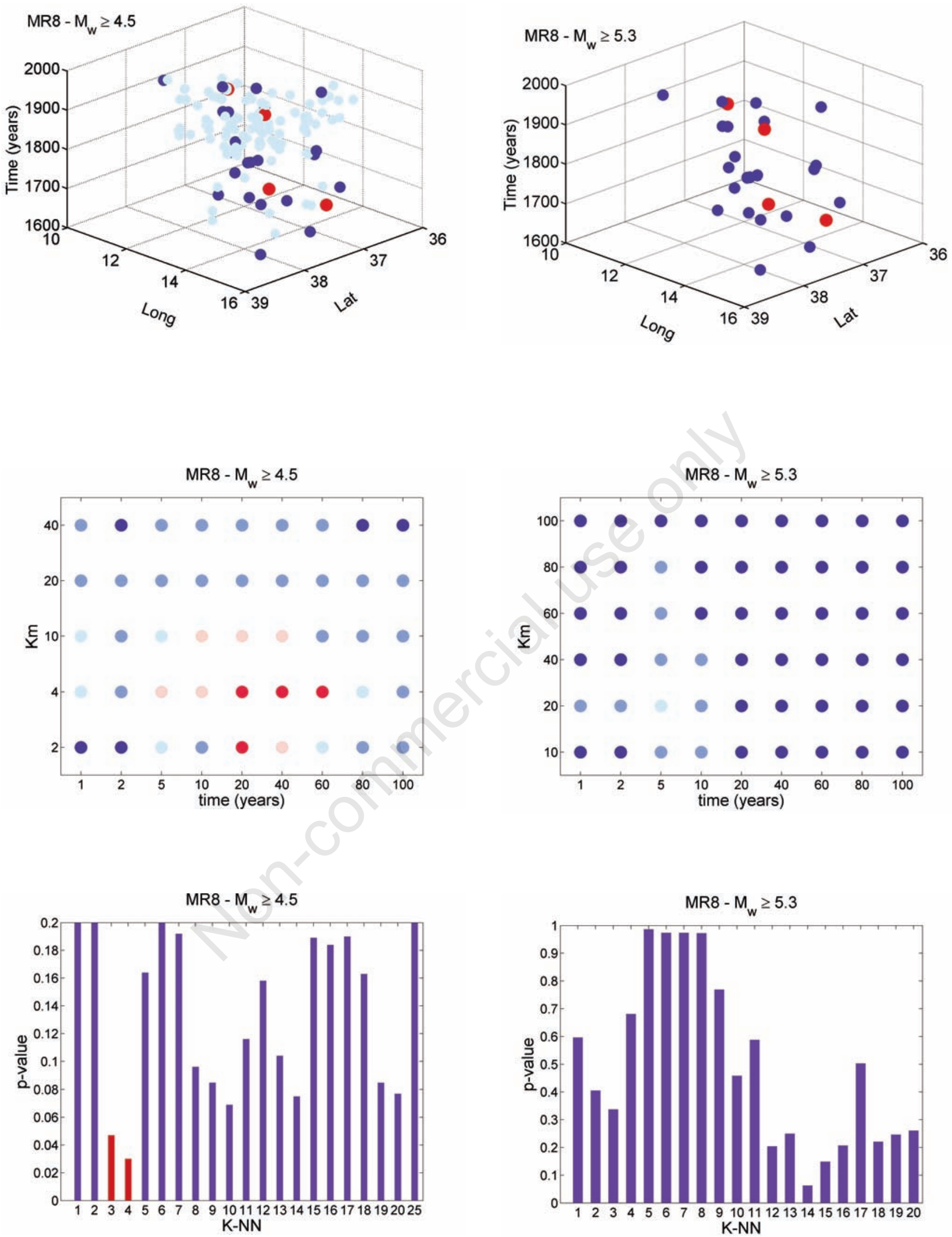

Figure 9. Results related to MR8 data with cut-off magnitude 4.5 (left) and 5.3 (right), respectively. See caption of Figure 2. MR, macroregions. 
Global tests enable us to select the data sets with significant spacetime clustering in order to perform deeper analyses for the detection of cluster locations and, possibly, for a better understanding of the fault interactions. Local tests could be primary statistical tools to this end; they could be applied to all the data with threshold magnitude 4.5 drawn from the macroregions. Further studies should be specifically planned for the macroregions MR2 and MR7 showing more decisive clustering trend.

\section{References}

1. Evison F, Rhoades D. Multiple-mainshock events and long-term 310 seismogenesis in Italy and New Zealand. New Zeal J Geol Geophys 2005;48:523-36.

2. Lombardi A, Marzocchi W. Evidence of clustering and nonstationarity in the time distribution of large worldwide earthquakes. J Geophys Res 2007;112:1-15.

3. Kagan YY, Jackson DD. Probabilistic forecasting of earthquakes. Geophys J Int 2000;143:438-53.

4. Kagan YY, Jackson DD. Global earthquake forecasts. Geophys J Int 2011;184:759-76.

5. International Commission on Earthquake Forecasting for Civil Protection. Operational earthquake forecasting. State of knowledge and guidelines for utilization. Ann Geophys 2011;54:315-91.

6. Pfeiffer D, Robinson T, Stevenson M, et al. Spatial analysis in epidemiology. Oxford, UK: Oxford University Press; 2008.

7. Tango T. Statistical methods for disease clustering. New York:
Springer; 2010.

8. Rogerson P, Yamada I. Statistical detection and surveillance of geographic clusters. London: Chapman \& Hall/CRC Interdisciplinary Statistics Series; 2009.

9. Gruppo di Lavoro CPTI. Catalogo Parametrico dei Terremoti Italiani, versione 2004 (CPTI04). Bologna, Italy: INGV; 2004. Available from: http://emidius.mi.ingv.it/CPTI04/

10. Basili R, Valensise G, Vannoli P, et al. The Database of Individual Seismogenic Sources (DISS), version 3: summarizing 20 years of research on Italy's earthquake geology. Tectonophysics 2008;453:2043.

11. Meletti C, Galadini F, Valensise G, et al. A seismic source zone model for the seismic hazard assessment of the Italian territory. Tectonophysics 2008;450:85-108.

12. David FN, Barton DE. Two space-time interaction tests for epidemicity. Br J Prev Soc Med 1966;20:44-8.

13. Knox G. The detection of space-time interactions. Appl Stat 1964;13:25-9.

14. Jacquez GM. A k nearest neighbour test for space-time interaction. Stat Med 1996;15:1935-49.

15. Wells DL, Coppersmith KJ. New empirical relationships among magnitude, rupture length, rupture width, rupture area, and surface displacement. Bull Seismol Soc Am 1994;84:974-1002.

16. MPS Working Group. Redazione della mappa di pericolosità sismica prevista dall'Ordinanza PCM 3274 del 20 marzo 2003. Rapporto Conclusivo per il Dipartimento della Protezione Civile. MilanoRoma, Italy: INGV; 2004. Available from: http://zonesismiche.mi. ingv.it 